\title{
(2) OPEN ACCESS \\ Do people reduce compliance with COVID-19 guidelines following vaccination? A longitudinal analysis of matched UK adults
}

\author{
Liam Wright (10 , ${ }^{1}$ Andrew Steptoe, ${ }^{2}$ Hei Wan Mak, ${ }^{2}$ Daisy Fancourt (ㄱ) ${ }^{2}$
}

\begin{abstract}
- Additional supplemental material is published online only. To view, please visit the journal online (http://dx doi.org/10.1136/jech-2021217179).
\end{abstract}

${ }^{1}$ Institute of Education, University College London, London, UK

'Department of Behavioural Science and Health, University College London, London, UK

\section{Correspondence to} Liam Wright, Institute of Education, University College London, London WC1H OAL, UK liam.wright.17@ucl.ac.uk

Received 3 May 2021 Accepted 22 June 2021
Check for updates

(C) Author(s) (or their employer(s)) 2021. Re-use permitted under CC BY. Published by BMJ.

To cite: Wright $L$, Steptoe $A$, Mak HW, et al. J Epidemiol Community Health Epub ahead of print: [please include Day Month Year]. doi:10.1136/jech-2021217179

\section{ABSTRACT}

Introduction COVID-19 vaccines do not confer immediate immunity and vaccinated individuals may still be at risk of transmitting the virus. Governments have not exempted vaccinated individuals from behavioural measures to reduce the spread of COVID-19, such as practising social distancing. However, vaccinated individuals may have reduced compliance with these measures, given lower perceived risks.

Methods We used monthly panel data from October 2020 to March 2021 in the UK COVID-19 Social Study to assess changes in compliance following vaccination. Compliance was measured with two items on compliance with guidelines in general and compliance with social distancing. We used matching to create comparable groups of individuals by month of vaccination (January, February or not vaccinated by February) and fixed effects regression to estimate changes in compliance over the study period.

Results Compliance increased between October 2020 and March 2021, regardless of vaccination status or month of vaccination. There was no clear evidence that vaccinated individuals decreased compliance relative to those who were not yet vaccinated.

Conclusion There was little evidence that sample members vaccinated in January or February reduced compliance after receiving vaccination for COVID-19. Continued monitoring is required as younger individuals receive the vaccine, lockdown restrictions are lifted and individuals receive second doses of the vaccine.

\section{INTRODUCTION}

Governments have begun mass vaccination programmes for COVID-19, but it will be several months before herd immunity is achieved. The available vaccines do not confer immediate immunity and are not $100 \%$ effective. ${ }^{1}$ Vaccinated individuals may still be at risk of catching and transmitting the virus, including variants they have not been inoculated against. ${ }^{2}$ Given this, the UK government has not exempted vaccinated individuals from behavioural measures to reduce the spread of COVID-19, such as the wearing of masks, practising social distancing and reducing household mixing.

International data show that, though compliance levels are high overall, not all individuals comply with recommended or mandated behavioural measures. ${ }^{3}$ While compliance has increased as countries have experienced second waves, overall compliance has decreased somewhat since the start of the pandemic. ${ }^{4}$ Vaccinated individuals, in particular, may feel less motivated to comply, given perceived lower health risks. Empirical evidence from the COVID-19 and previous epidemics, ${ }^{5-7}$ and predictions from influential models of health behaviour, such as the Risk Compensation, Health Belief and COM-B models, ${ }^{8-10}$ suggest that individuals who are less concerned about catching a virus have lower compliance. Further, in the UK, citizens have expressed difficultly keeping abreast of latest rules, ${ }^{11-14}$ due to variations in rules across areas and over time and (speculatively) due to 'lockdown fatigue'. Vaccinated individuals may therefore not be aware of non-exemption from government rules.

Early evidence from vaccine roll-out in Israel and the UK finds some increase in infection rates following first vaccination, ${ }^{15}{ }^{16}$ and infection rates have risen in Chile despite high vaccination rates. ${ }^{17}$ Some have argued that this may reflect lower compliance with protective behaviours. ${ }^{18-20}$ This is supported by survey evidence from early December 2020 that $40 \%$ of UK respondents intended to comply less or not comply with government guidelines following vaccination ${ }^{21}$ and with recent evidence that a sizeable minority of vaccinated over 80 s in the UK have subsequently broken household mixing rules. ${ }^{22}$ Further, longitudinal evidence from influenza and Lyme's disease vaccination programmes shows reduced compliance with some protective behaviours. ${ }^{23}$ Yet, crosssectional evidence inquiring about changes in behaviour following COVID-19 vaccination shows more over-80s reporting increased compliance (8\%-15\%) with hand-washing, face mask wearing and social distancing rules than decreased compliance $(1 \%-2 \%) .^{22}$

Given the risk of vaccinated individuals catching and transmitting the virus, understanding whether people comply less following vaccination is important for managing the pandemic. ${ }^{25}$ Yet, there is a notable lack of rigorous research on the consequences of COVID-19 vaccination for personal protective behaviours. ${ }^{20}$ Therefore, in this paper, we used monthly panel data from a large sample of UK adults to explore changes in compliance following vaccination.

\section{METHODS \\ Sample}

Data were drawn from the COVID-19 Social Study; a large ongoing panel study of the psychological and social experiences of over 70000 adults (aged 
$18+)$ in the UK during the COVID-19 pandemic. The study commenced on 21 March 2020 and involves online weekly (from August 2020, monthly) data collection from participants for the duration of the COVID-19 pandemic in the UK. The study is not random and therefore is not representative of the UK population, but it does contain a heterogeneous sample. Participants were recruited using three primary approaches. First, convenience sampling was used, including promoting the study through existing networks and mailing lists (including large databases of adults who had previously consented to be involved in health research across the UK), print and digital media coverage, and social media. Second, more targeted recruitment was undertaken focusing on (1) individuals from a low-income background, (2) individuals with no or few educational qualifications and (3) individuals who were unemployed. Third, the study was promoted via partnerships with third sector organisations to vulnerable groups, including adults with pre-existing mental health conditions, older adults, carers and people experiencing domestic violence or abuse.

For these analyses, we focused on participants aged 89 or younger who completed the monthly survey in each of the 6 months between 23 September 2020 and 22 March 2021 $(\mathrm{n}=23287 ; 62.3 \%$ of individuals with data collection between these dates; $32.6 \%$ interviewed at any point). Ages are capped at age 90 in the data, so we excluded participants aged 90 or above from this analysis. Though there is slight overlap in calendar months, for brevity, below we refer to the survey waves as October, November, December, January, February and March waves, respectively. We used matching in this analysis and excluded participants with missing data on any variable used $(\mathrm{n}=827 ; 3.6 \%$ of the eligible sample). This left a total sample size of 22460 .

The vaccine roll-out began in the UK on 8 December 2020. Seven hundred sixty-eight thousand individuals were vaccinated in England by 27 December 2020, 6.3 million by 28 January 2020 and 14.9 million by 25 February 2020 (1.4\%, 11.4\%, $27.0 \%$ of the population, respectively). ${ }^{26}$ The COVID-19 Social Study does not contain information on the date of vaccination, but given few individuals reported being vaccinated on, or shortly after, 23 December 2020, we assume that no participants were vaccinated before this date $(1.32 \%$ of participants recorded vaccination on 23 December 2020). The vaccine was initially rolled out in age order, beginning with over 80 year olds, then over $75 \mathrm{~s}$ and over $70 \mathrm{~s}$. Frontline health and social care workers, older adults in care homes and clinically extremely vulnerable individuals were also offered the vaccine. ${ }^{27}$

The period studied here coincides with the second wave of COVID-19 in the UK. There have been a several changes to government rules across this period. Online supplemental figure S1 displays the Oxford COVID-19 Government response tracker, ${ }^{28}$ a numeric summary of the severity of COVID-19 measures across time, along with death rates and new case rates of COVID-19. Changes to government policy are described further in the online supplemental information.

\section{Measures}

Compliance was measured with two questionnaire items, which we analysed separately. General compliance was measured with a single-item question, 'Are you following the recommendations from authorities to prevent the spread of COVID-19?'. Responses ranged from ' 1 . Not at all' to '7. Very much so'. Social distancing was measured with a single question 'When you go out or meet with others have you been maintaining social distancing?'. The responses categories ranged from ' 1 . Yes, completely' to ' 4 . Not at all' with an extra category for those who had not met with others or left their home in the last week. We reverse code this item so high scores indicate greater compliance and code those who did not leave them home or meet with others as the highest level of compliance (range 1-5).

\section{Statistical analysis}

Our analysis proceeded in three steps. First, we split our sample into three groups: individuals who first reported being vaccinated in the January wave; individuals who first reported being vaccinated in the February wave and individuals who did not report being vaccinated by February. Second, given the rules used for roll-out of the vaccine, we used matching to obtain samples of similar individuals across the three groups. As our 'treatment' variable (vaccination) had three levels, we carried out matching for each combination of two groups, obtaining three matched samples (January vs February vaccinators; February vs nonvaccinated and January vs non-vaccinated). Observations were matched using Mahalanobis distance within a calliper of $0.25 \mathrm{SD}$ in propensity scores. We used 1-to-1 matching without replacement and discarded observations outside the region of common support.

In the Mahalanobis distance step, given vaccine eligibility criteria set out by the UK Government, we matched on age, date of interview in the December wave, whether the participant was a keyworker, and whether they had a influenza vaccine in the past year (an indicator of existing health problems and willingness to accept vaccination). To estimate propensity scores, we used variables for age (natural splines with $\mathrm{df} 3$ ), date of data collection in December (natural splines with df 3), keyworker status, previous influenza vaccination, sex, general compliance and social distancing in the December wave (inputted as categorical variables), attitudes to vaccination (exploratory factor analysis of 12 items; September wave), intention to receive COVID-19 vaccination (September wave; categorical variable), whether the participant reported shielding for health reasons at any point, number of chronic health conditions $(0,1,2+)$ and whether the participant had a diagnosis for a psychiatric condition. More detail on these variables is given in the online supplemental information. We assessed match quality as bias $<0.1 \mathrm{SD}$ for each covariate, Rubin's B $<0.25$, Rubin's $\mathrm{R}$ of $0.5-5$, and visual inspection of the distributions for variables used in the Mahalanobis distance matching step.

In the third step, we estimated fixed effects regression models for each matched sample, separately, comparing within-person changes in compliance behaviour by wave of data collection across vaccination groups. Our model was of the form:

$$
\begin{aligned}
\text { Compliance }_{i t}= & \beta_{W} \cdot \text { Wave }_{i t}+\beta_{V} \cdot \text { Wave }_{i t} \cdot \text { Vaccinated }_{i}+ \\
& \beta_{T} \cdot \text { Date }_{i t}+\mu_{i}+\varepsilon_{i t}
\end{aligned}
$$

where $i$ and $t$ index individuals and waves, respectively. Wave $e_{i t}$ is a categorical variable for wave of data collection (December wave used as reference category). Vaccinated $_{i}$ is an indicator for vaccination group; $\beta_{V}$ is a vector of coefficients assessing differences in within-person changes in compliance by wave of data collection; Date $e_{i t}$ is a vector of date fixed effects to account for time trends in compliance behaviour; and $\mu_{i}$ and $\varepsilon_{i t}$ are personspecific and observation-specific random errors, respectively.

Our interest was in the sign and size of the coefficients $\beta_{V}$. Our hypothesis was that, compared with non-vaccinated individuals, compliance would be lower among vaccinated individuals in the months that they were vaccinated, and, given that vaccination does not confer immediate immunity, progressively lower the 
Table 1 Sample sizes in matched samples

\begin{tabular}{lllcc}
\hline Sample & $\mathbf{n}$ & Rubin's R & Rubin's B & Rubin's B (pairs) \\
\hline January vs February & 2004 & 1.11 & 7.97 & 11.93 \\
January vs not vaccinated & 1294 & 1.11 & 6.75 & 8.23 \\
February vs not vaccinated & 7596 & 1.20 & 15.16 & 17.09 \\
\hline
\end{tabular}

Success of the propensity score matching was assessed using Rubin's B $<25 \%$, Rubin's R of $0.5-2$ and a bias of $<10 \%$ SD for each covariate.

more time had elapsed since vaccination. There should also be no differences in compliance levels in the months prior to vaccination. In our data, this hypothesis translated into no differences in compliance by vaccination status in October, November and December; differences in compliance in January, February and March when comparing January vaccinators with February vaccinated or non-vaccinated individuals and differences in compliance in February and March but not January when comparing February vaccinators with non-vaccinated individuals. In short, if vaccines lead to a change in behaviour and if our matching procedure has worked well, differences in compliance should be observed after vaccination but not before. This is what we test with our regression models.

Data analysis was carried out in R V.4.0.3. ${ }^{29}$ Matchings was carried out using the matchit package. ${ }^{30}$ Due to stipulations set out by the ethics committee, data will be made available at the end of the pandemic. The code to replicate the analysis is available at https://osfio/xghvb/.

\section{RESULTS}

\section{Descriptive statistics}

Descriptive statistics for the full sample are displayed in online supplemental table S1. There were several differences among the vaccination groups, most notably on age, keyworker status and date of December interview. Differences were markedly smaller following matching (online supplemental table S2). Figures showing standardised mean differences in the study variables across matched and unmatched samples are displayed in online supplemental figures S2-S4. Matching reduced differences in almost all cases. In the January vs February and February vs nonvaccinated comparison groups, (absolute) standardised mean differences were less than $0.1 \mathrm{SD}$ in each case. The quality of the matching was lower in the January vs unvaccinated groups, though Rubin's B and R statistics were within boundaries considered to be acceptable matching (table 1). Online supplemental figures S5-S7 show the distributions of age, date of date collection in December and keyworker status in the matched samples, specifically, given these are important predictors of vaccination status. Matching in the January vs February vaccination comparison group was successful, but there were notable differences in the distributions of age and survey date in the January vs nonvaccinated and February vs non-vaccinated groups, respectively.

Figure 1 shows the trends in each compliance measure over the study period. As the UK entered a second wave, there were increases in both compliance measures, though with some decrease in social distancing over December. ${ }^{3} 31$

\section{Vaccinations and compliance behaviour}

The results of the fixed effects regressions are displayed in figure 2. There were no statistically significant differences in either compliance measure following vaccination in any matched sample group. There were also no statistical significant differences prior to vaccination, suggesting this in no biased by unobserved confounding in the matched samples.
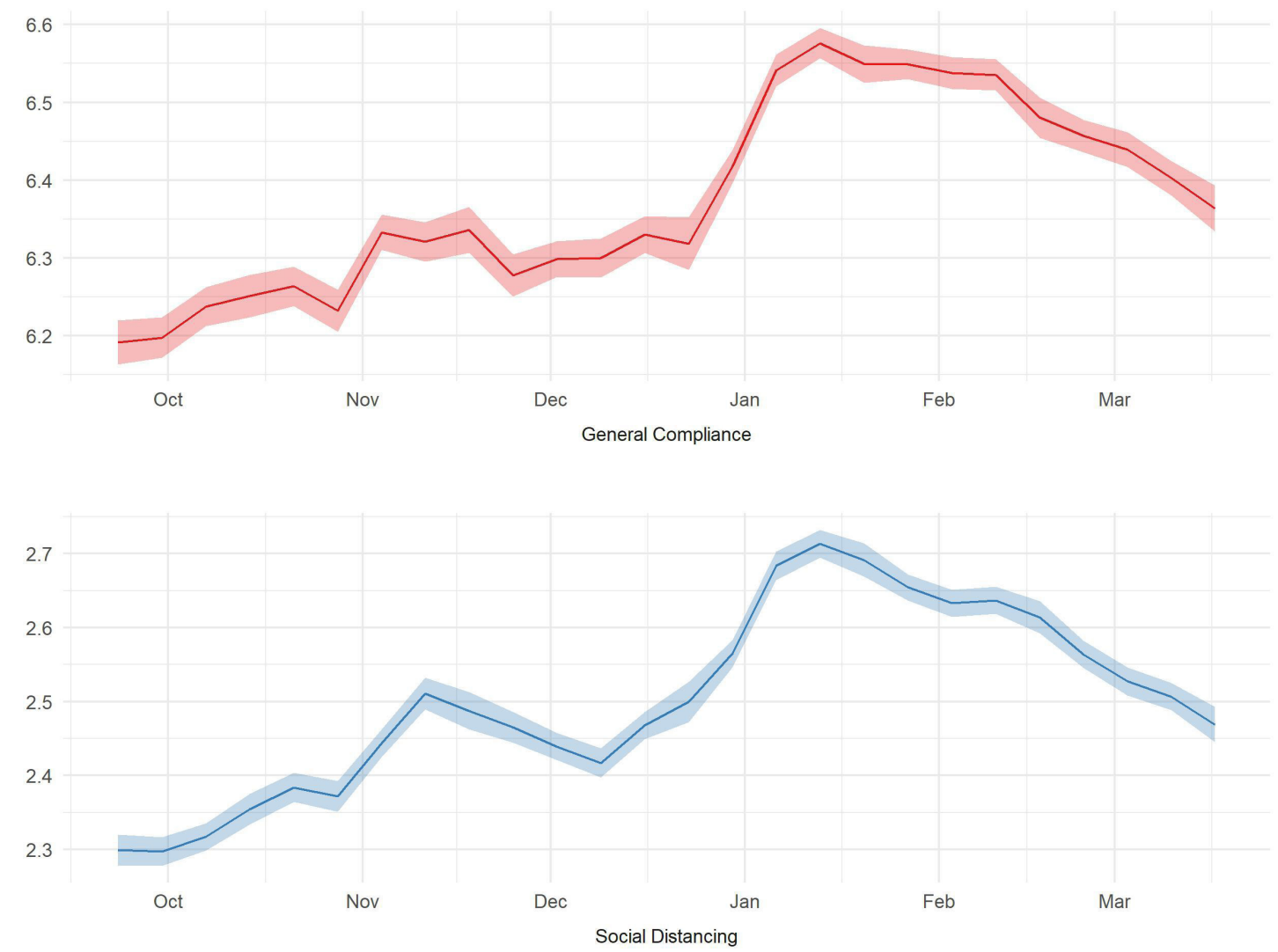

Figure 1 Trends in compliance behaviours. 


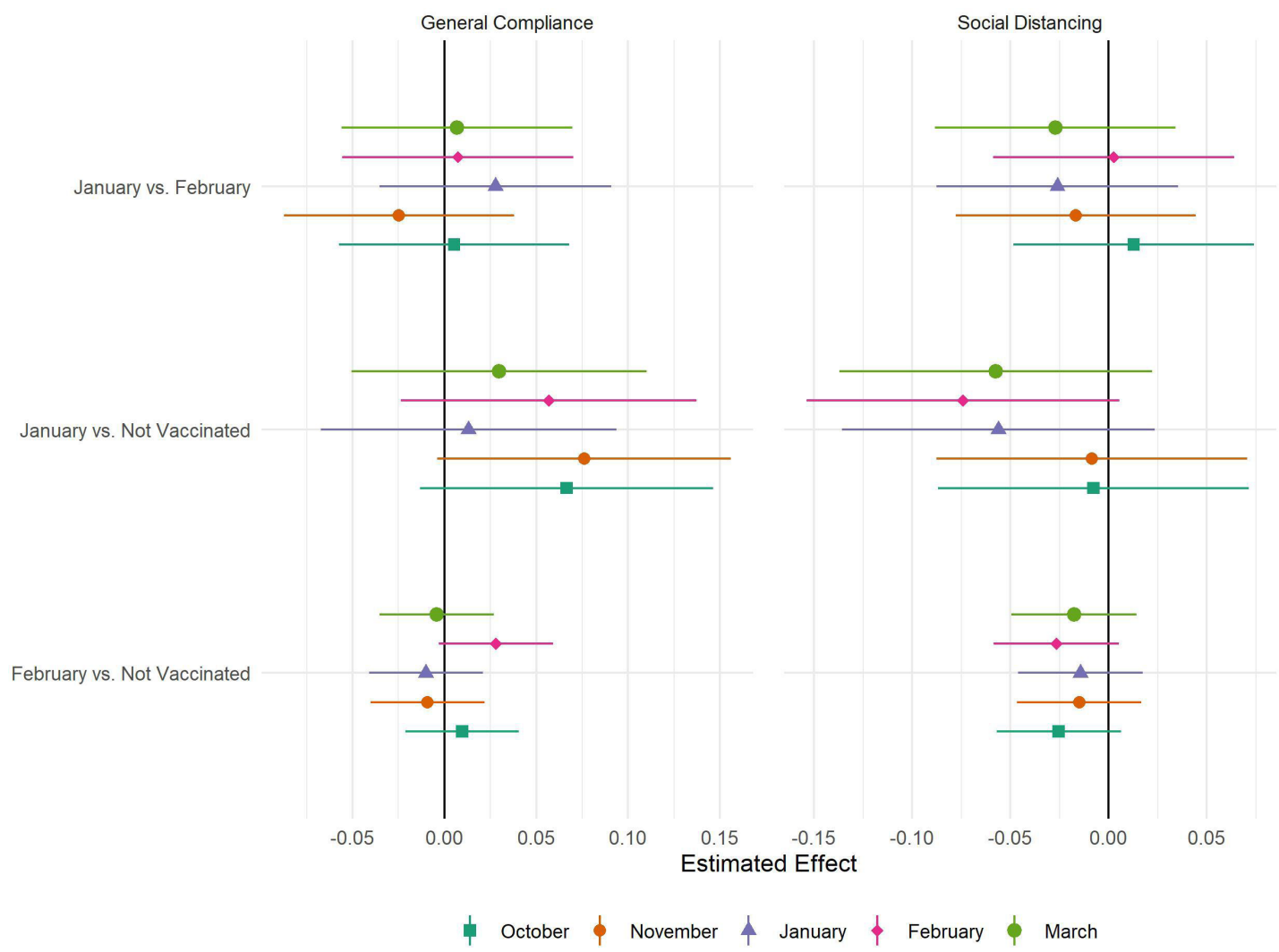

Figure 2 Results of fixed effects regression by matched sample and measure of compliance.

It is possible that small average differences may mask heterogeneous effects-a small number of vaccinated individuals could stop complying altogether. To explore this, figure 3 displays bar plots for compliance levels at each interview in the January vs February vaccination matched sample. There was no clear evidence of extremely low levels of compliance in the vaccinated group. The same is true when comparing February vaccinators or January vaccinators with non-vaccinated individuals (online supplemental figures S8 and S9). In fact, as shown in online supplemental table S2, average compliance levels increased among all groups between October and February in line with the increase in compliance seen in the wider population.

\section{Sensitivity analyses}

Given that fixed effects regressions compare within-person changes in compliance levels across vaccination groups, we also repeated the model in (1) using mixed effects modelling, interpreting the term $u_{i}$ as a normally distributed random intercept. These regressions tested differences in compliance levels by vaccination status and wave. The results are shown in online supplemental figure S10 and are qualitatively similar to those shows in figure 2 .

\section{DISCUSSION}

Using panel data from 5 months of the pandemic in the UK, we found no clear evidence that receiving a COVID-19 vaccine reduced compliance behaviour. Descriptively, there was little evidence of vaccinated individuals reducing compliance altogether. In fact, vaccinated individuals-like non-vaccinated individuals - increased compliance from the beginning of the period as the UK experienced its second wave of COVID-19.

The results are striking given existing evidence that compliance levels are higher among those with greater health risks from-or greater fears of-catching COVID- $19,{ }^{57}$ and evidence of widespread intentions to reduce compliance following vaccination. ${ }^{21}$ An explanation for the discrepancy may be the almost exclusive use of cross-sectional data in the literature-a recent study shows that marked differences in between-person and within-person associations between compliance and several factors. ${ }^{32}$ The results suggest that vaccinations do not crowd-out other preventive behaviours. However, it should be noted that we used a relatively short follow-up period-differences in compliance may take time to arise, especially as individuals are warned that vaccines do not take effect immediately and second vaccinations are required for full effectiveness. Vaccinated individuals in our sample were also relatively old. The results may have been different were vaccinations rolled out more widely - intentions to reduce compliance or not comply following vaccination are higher among younger age groups. ${ }^{21}$ Further, compliance was measured during a period of strict lockdown where the opportunities for non-compliance were limited. This study should be repeated as lockdowns are eased. We also only focused on two measures of compliance. Differences could potentially be observed for other behaviours, such as indoor or outdoor household mixing.

This study had a number of other limitations. First, we used two self-report measures of compliance which may be subject to biases such as recall bias or social desirability bias. Being vaccinated could be considered a form of compliance so our general compliance measure may not have been specific enough to pick up on differences in other behaviours. Further, this measure does not clarify which rules individuals are violating when not complying, such as those regarding face masks or household mixing. The social distancing measure also encapsulates multiple behaviours, such as maintaining space from members of the public in shops or on pavements and limiting close contact with family and friends in indoor or outdoor locations. 

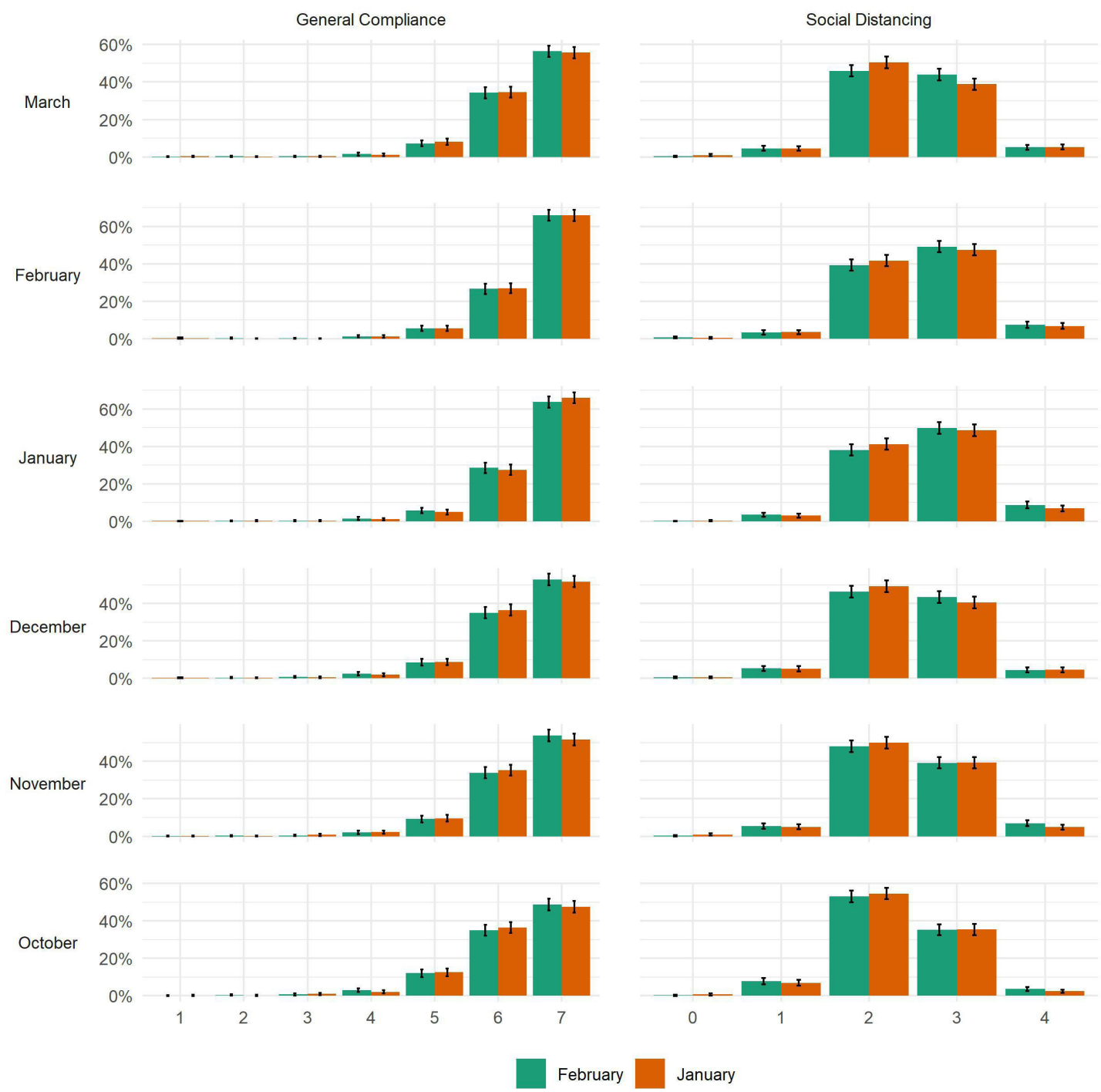

Figure 3 Distribution of compliance behaviours by vaccination status and wave, January vs February vaccinated matched sample.

Second, our sample was not representative and, moreover, was comprised of individuals who comply more than on average. ${ }^{33} \mathrm{~A}$ disproportionate number of our sample may have been motivated to comply by concerns other than personal risk (eg, acting out of civic duty) and so exhibit smaller changes in compliance following vaccination than would be observed in the general population. An issue with examining changes in compliance using survey data is that the characteristics that determine willingness to participate in a survey may overlap with those relate to willingness to comply with guidelines. Another approach for examining behaviour change following vaccination may be to use passively collected data, such as that on mobility using smartphone location data.

Third, the existence of the vaccine programme may have induced behaviour changes in the non-vaccinated group, if these individuals were less concerned about transmitting the virus. ${ }^{34}$ Fourth, compliance was changing over time, even in the absence of vaccination. Previous research has shown that the strength of several factors in predicting compliance differs over pandemics. ${ }^{33}$ Our matched samples may therefore not provide an appropriate counterfactual and results may be biased by unobserved confounding. Nevertheless, by exploiting the longitudinal nature of our sample, we were able to use compliance in months prior to vaccination as a placebo test. No statistically significant differences were found in these months, which may add confidence to our results.
Our results suggest that there is no immediate cause for concern of widespread non-compliance among vaccinated individuals. However, it is important to continue monitoring the situation as the vaccine is roll-out more widely, restrictions are lifted and people receive second doses. Analyses using data from other populations and that examine the potential impact of widespread vaccination on the behaviour of those not yet vaccinated are also required in order to ensure that the gains of the vaccination programme are not lost through increases in risky behaviour.

\section{What is already known on this subject}

COVID-19 vaccines do not confer immediate immunity and vaccinated individuals may still be at risk of transmitting the virus. Governments have not exempt vaccinated individuals from other preventive measures, such as social distancing.

- Survey evidence suggests that many individuals intend to reduce compliance with COVID-19 guidelines following vaccination, but to our knowledge there have been no direct tests of vaccinations leading to reducing compliance during COVID-19 to date. 


\section{What this study adds}

- We found some evidence of reduced compliance with social distancing following vaccination, relative to compliance levels observed among non-vaccinated individuals, but this was not replicated in all samples.

- We also found that vaccinated individuals actually increased compliance over the study period, a period that overlapped with the second wave of COVID-19 in the UK.

- The results suggest that individuals do not substantially decrease compliance following vaccination.

- However, this analysis requires replication with general population data, as individuals receive second doses of the vaccine, as lockdowns are eased and as younger individuals begin to be vaccinated.

Acknowledgements The researchers are grateful for the support of a number of organisations with their recruitment efforts including: the UKRI Mental Health Networks, Find Out Now, UCL BioResource, HealthWise Wales, SEO Works, FieldworkHub and Optimal Workshop.

Contributors LW, AS, HWM and DF conceived and designed the study. LW analysed the data and wrote the first draft. All authors provided critical revisions.

Funding This COVID-19 Social Study was funded by the Nuffield Foundation (WEL/FR-000022583), but the views expressed are those of the authors and not necessarily the Foundation. The study was also supported by the MARCH Mental Health Network funded by the Cross-Disciplinary Mental Health Network Plus initiative supported by UK Research and Innovation (ES/S002588/1) and by the Wellcome Trust (221400/Z/20/Z). DF was funded by the Wellcome Trust (205407/Z/16/Z). The study was also supported by HealthWise Wales, the Health and Car Research Wales initiative, which is led by Cardiff University in collaboration with SAlL, Swansea University.

Disclaimer The funders had no final role in the study design; in the collection, analysis and interpretation of data; in the writing of the report or in the decision to submit the paper for publication. All researchers listed as authors are independent from the funders and all final decisions about the research were taken by the investigators and were unrestricted.

\section{Competing interests None declared.}

Patient consent for publication Not required.

Ethics approval The study was approved by the UCL Research Ethics Committee (12467/005) and all participants gave informed consent. The study protocol and user guide (which includes full details on recruitment, retention, data cleaning and sample demographics) are available at https://githubcom/UCLBSH/CSSUserGuide.

Provenance and peer review Not commissioned; externally peer reviewed.

Data availability statement No data are available. Data used in this study will be made publicly available once the pandemic is over. The code used to run the analysis is available at https://osf.io/xghvb/.

Supplemental material This content has been supplied by the author(s). It has not been vetted by BMJ Publishing Group Limited (BMJ) and may not have been peer-reviewed. Any opinions or recommendations discussed are solely those of the author(s) and are not endorsed by BMJ. BMJ disclaims all liability and responsibility arising from any reliance placed on the content. Where the content includes any translated material, BMJ does not warrant the accuracy and reliability of the translations (including but not limited to local regulations, clinical guidelines, terminology, drug names and drug dosages), and is not responsible for any error and/or omissions arising from translation and adaptation or otherwise.

Open access This is an open access article distributed in accordance with the Creative Commons Attribution 4.0 Unported (CC BY 4.0) license, which permits others to copy, redistribute, remix, transform and build upon this work for any purpose, provided the original work is properly cited, a link to the licence is given, and indication of whether changes were made. See: https://creativecommons.org/ licenses/by/4.0\%

\section{ORCID iDs}

Liam Wright http://orcid.org/0000-0002-6347-5121

Daisy Fancourt http://orcid.org/0000-0002-6952-334X

\section{REFERENCES}

1 Public Health England. Public Health England vaccine effectiveness report: March 2021, 2021. Available: https://www.gov.uk/government/publications/phe-monitoringof-the-effectiveness-of-covid-19-vaccination

2 European Centre for Disease Prevention and Control. Risk of SARS-CoV-2 transmission from newly-infected individuals with documented previous infection or vaccination. Stockholm, 2021.

3 YouGov. Personal measures taken to avoid COVID-19, 2021. Available: https://yougov. co.uk/topics/international/articles-reports/2020/03/17/personal-measures-takenavoid-covid-19 [Accessed 21 Sep 2020].

4 Petherick A, Goldszmidt R, Andrade EB. A worldwide assessment of COVID-19 Pandemic-Policy fatigue. Rochester, NY: Social Science Research Network, 2021

5 Barber SJ, Kim H. COVID-19 worries and behavior changes in older and younger men and women. J Gerontol B Psychol Sci Soc Sci 2021;76:gbaa068:e17-23.

6 Bish A, Michie S. Demographic and attitudinal determinants of protective behaviours during a pandemic: a review. Br J Health Psychol 2010;15:797-824.

7 Harper CA, Satchell LP, Fido D, et al. Functional fear predicts public health compliance in the COVID-19 pandemic. Int J Ment Health Addict 2020:1-14.

8 Michie S, van Stralen MM, West R. The behaviour change wheel: a new method for characterising and designing behaviour change interventions. Implement Sci 2011;6:42.

9 Peltzman $S$. The effects of automobile safety regulation. J Polit Econ 1975:83:677-725.

10 Rosenstock IM, Strecher VJ, Becker MH. Social learning theory and the health belief model. Health Educ Q 1988;15:175-83.

11 Denford S, Morton KS, Lambert H. Understanding patterns of adherence to COVID-19 mitigation measures: a qualitative interview study. medRxiv 2020.

12 Williams SN, Armitage CJ, Tampe T, et al. Public perceptions and experiences of social distancing and social isolation during the COVID-19 pandemic: a UK-based focus group study. BMJ Open 2020;10:e039334.

13 Williams SN, Armitage CJ, Tampe T. Public perceptions of non-adherence to COVID-19 measures by self and others in the United Kingdom. medRxiv 2020.

14 Wright L, Burton A, McKinlay A. Public opinion about the UK government during COVID-19 and implications for public health: a topic modelling analysis of openended survey response data. Public Global Health 2021.

15 Bernal JL, Andrews N, Gower C. Early effectiveness of COVID-19 vaccination with BNT162b2 mRNA vaccine and ChAdOx1 adenovirus vector vaccine on symptomatic disease, hospitalisations and mortality in older adults in England. medRxiv 2021.

16 Hunter PR, Brainard J. Estimating the effectiveness of the Pfizer COVID-19 BNT162b2 vaccine after a single dose. A reanalysis of a study of 'real-world' vaccination outcomes from Israel. medRxiv 2021.

17 Mander B, Stott M. Chile's lauded vaccine rollout fails to save it from Covid surge, 2021. Financial Times. Available: https://www.ft.com/content/89992bc9-051a-42b58be0-60edad4165cb [Accessed 12 Apr 2021].

18 Independent SAGE. Maintaining adherence to protective behaviours during vaccination roll-out: summary and recommendations, 2021. Available: https://www.independentsage. org/wp-content/uploads/2021/01/Adverse-behavioural-effects-of-vaccines-7.1.pdf

19 et alRubin GJ, Brainard J, Hunter P. Are people letting down their guard too soon after covid-19 vaccination? 2021. BMJ Opinion. Available: https://blogs.bmj.com/bmj/2021/ 03/18/are-people-letting-down-their-guard-too-soon-after-covid-19-vaccination/ [Accessed 8 Apr 2021].

20 Scientific Pandemic Insights Group on Behaviours. SPI-B: Possible impact of the COVID-19 vaccination programme on adherence to rules and guidance about personal protective behaviours aimed at preventing spread of the virus, 17 December 2020, 2021. Available: https://www.gov.uk/government/publications/spi-b-possibleimpact-of-the-covid-19-vaccination-programme-on-adherence-to-rules-and-guidanceabout-personal-protective-behaviours-aimed-at-preventi [Accessed 7 Apr 2021].

21 YouGov. YouGov / sky survey results, 2020. Available: https://docs.cdn.yougov.com/ 8jj48aj08c/SKY_Vaccine_201203.pdf

22 ONS. Coronavirus and vaccine attitudes and behaviours in England: over 80s population, 15 February to 20 February 2021, 2021. Available: https://www.ons. gov.uk/peoplepopulationandcommunity/healthandsocialcare/conditionsanddiseases/ bulletins/coronavirusandvaccineattitudesandbehavioursinengland/over80spopulatio n15februaryto20february2021\#behaviours-of-over-80s-after-receiving-at-least-onedose-of-a-coronavirus-covid-19-vaccine [Accessed 8 Apr 2021].

23 Brewer NT, Cuite CL, Herrington JE, et al. Risk compensation and vaccination: can getting vaccinated cause people to engage in risky behaviors? Ann Behav Med 2007:34:95-9

24 Reiber C, Shattuck EC, Fiore $S$, et al. Change in human social behavior in response to a common vaccine. Ann Epidemiol 2010;20:729-33.

25 Ioannidis JPA. Benefit of COVID-19 vaccination accounting for potential risk compensation. Rochester, NY: Social Science Research Network, 2021.

26 NHS England. COVID-19 vaccinations, 2021. Available: https://www.england.nhs.uk/ statistics/statistical-work-areas/covid-19-vaccinations/ [Accessed 4 Feb 2021].

27 Public Health England. Why you have to wait for your COVID-19 vaccine, 2021. Available: https://www.gov.uk/government/publications/covid-19-vaccinationwhy-you-are-being-asked-to-wait/why-you-have-to-wait-for-your-covid-19-vaccine [Accessed 4 Feb 2021]. 
28 Hale T, Angrist N, Cameron-Blake E. Oxford COVID-19 government response Tracker. Oxford: Blavatnik School of Government, 2020. https://www.bsg.ox.ac.uk/research/ research-projects/coronavirus-government-response-tracker

29 R Core Team. R: a language and environment for statistical computing. Vienna, Austria: R Foundation for Statistical Computing, 2020. https://www.R-project.org/

30 Ho DE, Imai K, King G, et al. Matchlt : Nonparametric Preprocessing for Parametric Causal Inference. J Stat Softw 2011;42:1-28.

31 Ipsos M. Britons increasingly abiding by the COVID-19 rules, with social responsibility and the NHS the primary drivers, 2020. Available: https://www.ipsos.com/ipsos-mori/ en-uk/britons-increasingly-abiding-covid-19-rules-social-responsibility-and-nhsprimary-drivers [Accessed 25 Feb 2021].
32 Wright L, Steptoe A, Fancourt D. Predictors of self-reported adherence to COVID-19 guidelines. A longitudinal observational study of 51,600 UK adults. Lancet Reg Health Eur 2021;4:100061.

33 Wright L, Fancourt D. Do predictors of adherence to pandemic guidelines change over time? a panel study of 21, 000 UK adults during the COVID-19 pandemic. medRxiv 2020.

34 Trogen B, Caplan A. Risk compensation and COVID-19 vaccines. Ann Intern Med 2021;174:858-9.

35 van der Weerd W, Timmermans DR, Beaujean DJ, et al. Monitoring the level of government trust, risk perception and intention of the general public to adopt protective measures during the influenza A (H1N1) pandemic in the Netherlands. BMC Public Health 2011;11:575. 